\title{
Cloud Security Tracking, Log Maintenance and Notification System for Multitenant Mission Critical Cloud Applications
}

\author{
Miss. Pooja Rajendra Vyawhare ${ }^{1}$, Miss. V. B. Bhagat ${ }^{2}$ \\ ${ }^{I}$ (Department of computer science and Engg, P. R. Pote (Patil) College of Engineering, Amravati, Maharashtra, \\ India) \\ ${ }_{2}^{2}$ (Department computer science and Engg, P. R. Pote (Patil) College of Engineering, Amravati, Maharashtra, \\ India)
}

\begin{abstract}
Cloud computing is a set of IT services that are provided to a customer over a network on a leased basis and with the ability to scale up or down their service requirements. It advantages to mention but a few include scalability, resilience, flexibility, efficiency and outsourcing non-core activities. Cloud computing offers an innovative business model for organizations to adopt IT services without upfront investment. Despite the potential gains achieved from the cloud computing, the organizations are slow in accepting it due to security issues and challenges associated with it. Security is one of the major issues which hamper the growth of cloud. The idea of handing over important data to another company is worrisome; such that the consumers need to be vigilant in understanding the risks of data breaches in this new environment. This paper introduces a detailed analysis of the cloud computing security issues and challenges focusing on the cloud computing types and the service delivery types.
\end{abstract}

Keywords: Cloud Computing, Cloud Security, Service Provider, SaaS.

\section{INTRODUCTION}

Multi tenant cloud based and mission critical application having special measures to protect the application from the attacks and threats. Some of them will be like Login tracking, Log maintenance, Transaction notification through SMS and email ,Account locking system, Better session and history management, Password management.

Banking Management System thus ensures smooth operation of the Real-Estate management tasks as well as keep the information about the employees and their salary. Bank is the place where customers feel the sense of safety for their property.

\section{Cloud Computing}

The word cloud is used as a metaphor for the Internet, based on the standardized use of a cloud-like shape to denote a network on telephony schematics and later to depict the Internet in computer network diagrams as an abstraction of the underlying infrastructure it represents. The cloud symbol was used to represent the Internet as early as 1994. Cloud Computing is a technology that uses the internet and central remote servers to maintain data and applications. Cloud computing allows consumers and businesses to use applications without installation and access their personal files at any computer with internet access. This technology allows for much more efficient computing by centralizing data storage, processing and bandwidth. A simple example of cloud computing is Yahoo email, Gmail, or Hotmail etc. All you need is just an internet connection and you can start sending emails. There are 3 types of cloud computing:

SaaS-Software as a Service

PaaS-Platform as a Service

IaaS-Infrastructure as a Service

\section{Software As A Service (Saas) [3]}

In the SaaS model, cloud providers install and operate application software in the cloud and cloud users access the software from cloud clients. This type of cloud computing delivers a single application through the browser to thousands of customers using a multitenant architecture. The cloud users do not manage the cloud infrastructure and platform on which the application is running.

\section{Cloud Security - Service Provider Priorities}

$>$ Effectively meet the advertised SLA, while optimizing cloud resource utilization.

$>$ Offer tenants capabilities for self-service, and achieve scaling through automation and simplification.

$>$ Computing clouds are changing the whole IT, service industry, and global economy. Clearly, cloud computing demands ubiquity, efficiency, security, and trustworthiness 
$>$ Effective trust management, guaranteed security, user privacy, data integrity, mobility support, and copyright protection are crucial to the universal acceptance of cloud as a ubiquitous service.

Ensure confidentiality, integrity, and availability in a multi-tenant environment.

\section{Existing System}

In the existing system, each bank have their own server and own database so that maintenance cost is high. So sometimes performance get slowdown. Storage capacity problems may present there in the existing system so causes higher software and hardware cost. Better than paying for similar commercial software which alone may be justification for switching to cloud applications. We are switching to proposed due to the reasons:

- Higher maintenance cost.

- High cost of ownership.

- More resources required.

- Limitation in storage space.

Hardware Interfaces

\section{Experimental Setup}

- CPU: Pentium4

- Ram 1GB

Software Interfaces

1. Deployment Platform: Windows

2. Web Server: IIS

3. Development Technology

(Serverside): ASP.NET 4.0 4.Languages : C\#

5. Component Programming: DLL

6. Services Database technologies: MS SQL Server 2012,ADO.NET

7 .Web Development: XML,HTML, DHTML, Javascript, AJAX, JQuery

8.Development Tool: Visual Studio 2010

Our system is intended to develop an application which is multi tenant cloud based and mission critical. Cloud computing offers an innovative business model for organizations to adopt IT services without upfront investment. To develop a cloud based system that demonstrates tracking of activities, maintaining log of events and notifying the users about probable threats.

Our system has following feature:

- $\quad$ Reduced cost of ownership (COO) and increased Return on investment (ROI)

- Multitenant - pay per use

- Sharing of centralized resources

- Device and location independence

- $\quad$ Scalability and Elasticity

\section{Cloud based application}

\section{Modules}

There are various users in the cloud based application are as follows:

- Cloud Administrator

- Client Administrator

- Client Users.

Centralized database features denotes that on the cloud there is one administrator and one database for all the users. We can store large data on that centralized database. Multi-tenant users use the application at the same time.

\section{Administration Module}

Multiple tenants such as banks request cloud for services.If one the bank such as SBI wants the service of cloud then this bank have to send request to cloud for registration,then cloud verify the bank and approve it's request and can login it.Then this cloud provides number of services to the bank and also play important role in monitoring those services. Usage Log contains all the transaction and services used by the bank, all those information stored in usage log table.Then it calculates rent and generate report and depending upon the rent bank has to pay. 


\section{Tracking and Log maintenance module}

This monitors every activity and maintains log of events. If we have to send fund to the third party then third party has to register it's account first and then can perform further transaction.

If one of the bank does not pay the rent regularly hen cloud can lock it's account. If SBI is locked then all the branches of SBI are also locked and no user of SBI can log-in. This tracking keep all details when user log-in and log-out,all transaction information and so on.

\section{Notification}

Sent notification to the concerned users and suggest corrective measures. If invalid user try to access the account then this notification is updated to the database.

If one of the user using it's password over and over then administration gives notification to the user for changing password.If unautherised user try to access the account three or more times then it's entry automatically updated in threat table.

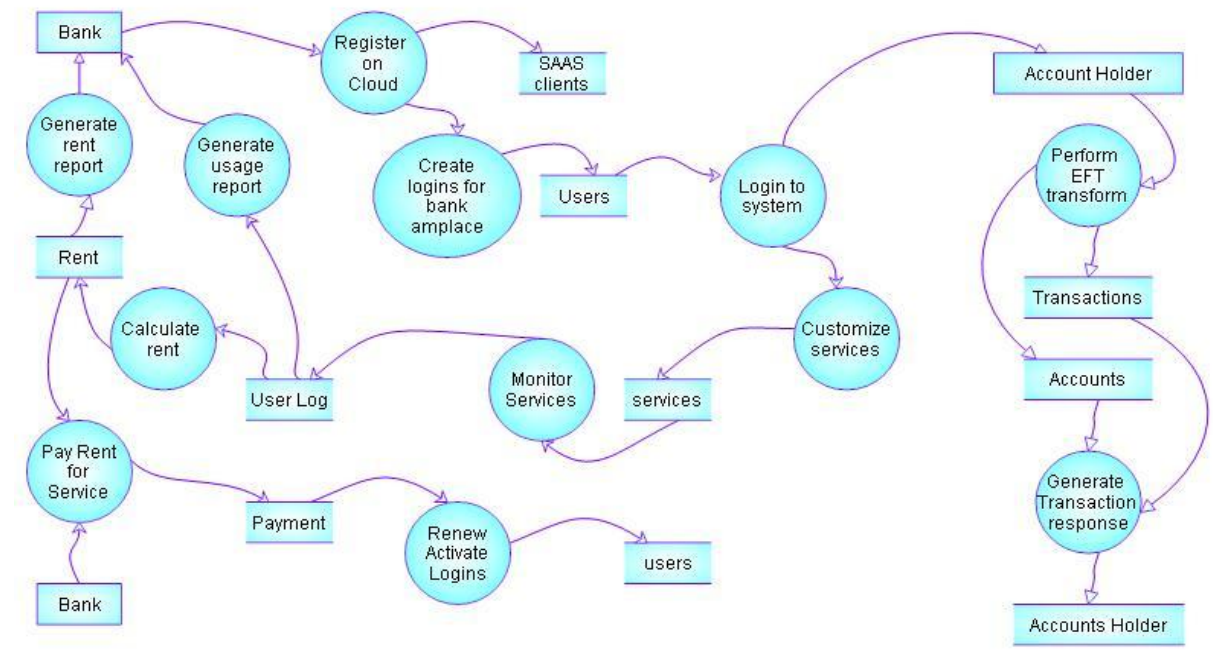

Fig 6.1: Transaction logins DFD

\section{Screenshots Of Gui Implementation}

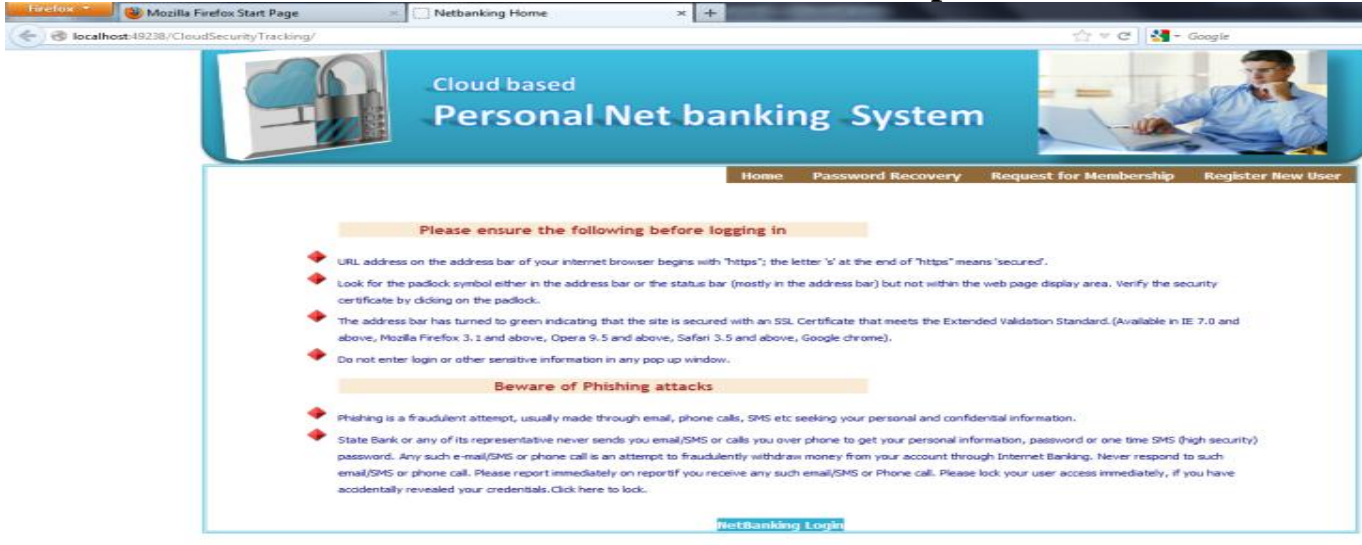

Screenshot No. 7.1: Home Page

This is Home Page of the Project .In this Page there are four link are available

1)Home 2) Password Recovery 3) Request for Membership 4) Register New User with the help of this link we have apply for membership as well as we also Registered new user. There is also one link available for login. 


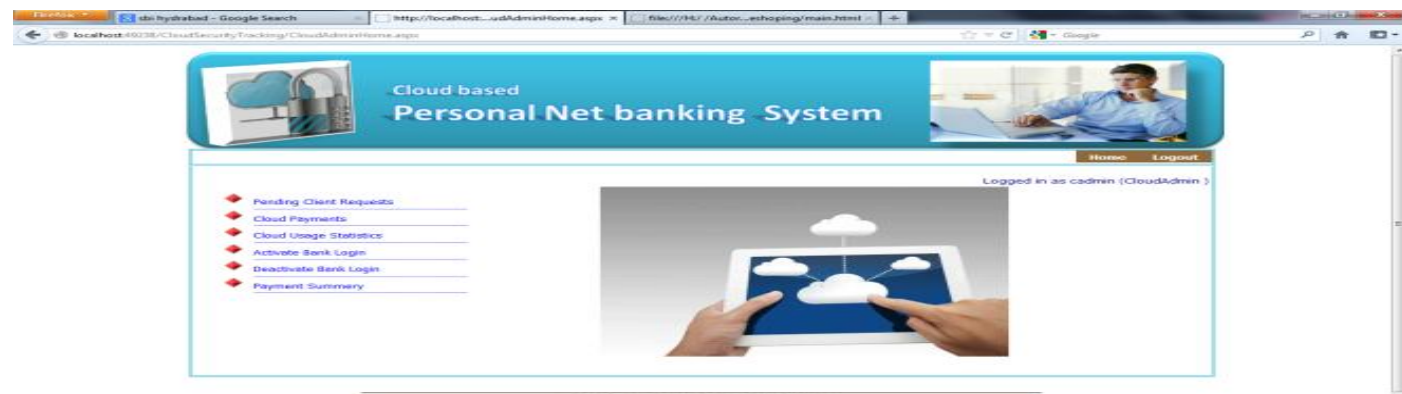

Screenshot No. 7.2: Cloud Admin Home Page

This is the home Page of cloud admin. He possesses six authorities.

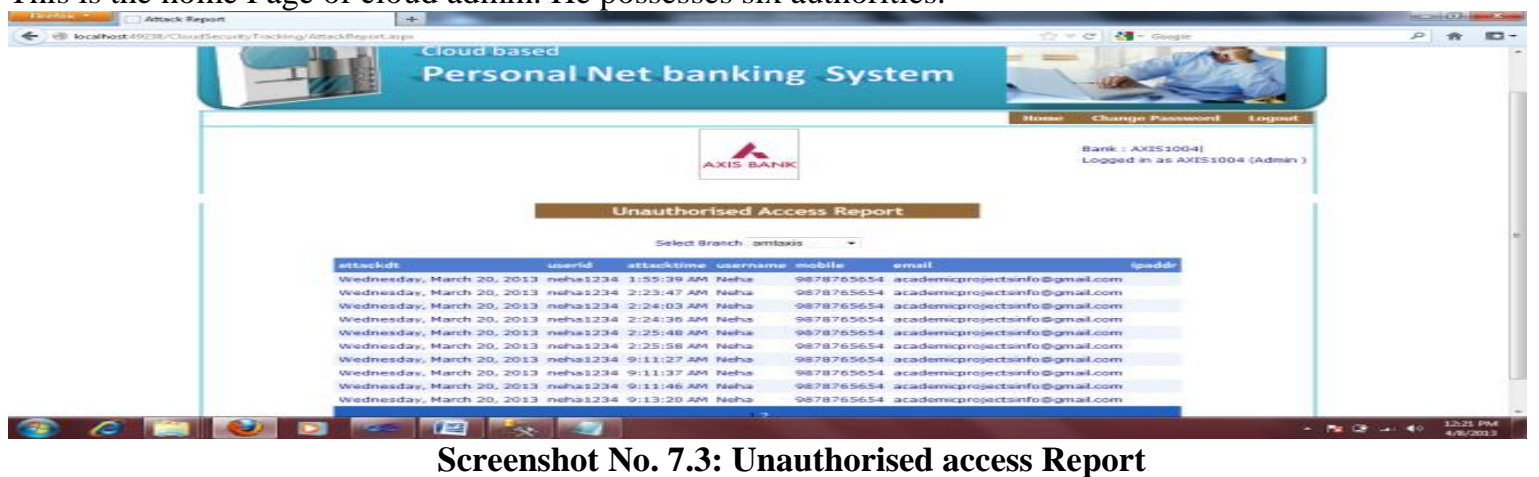

Unauthorized Access Report: - If people attempting to violet your security then this link maintaining the log with ipaddress for unauthorized users.

\section{Conclusion}

In cloud security tracking and notification system we use the cloud multitenant centralised database for storing net banking related information with various security constraints such as password mechanism for every user and administrator. Notification is given to user on behalf of security purpose. The main purpose of our application is to provide more security in net banking with the help of cloud.

\section{References}

[1]. Force.com, "The Force.com Multitenant Architecture Understanding the Design of Salesforce.com's Internet Application Development Platform", White paper

[2]. Qihong Shao "Towards Effective and Intelligent Multi-tenancy SaaS",UMI Dissertation publishing, May 2011

[3]. UNDERSTANDING The Cloud Computing Stack SaaS, Paas, IaaS, @ D Diversity Limited, 2011 Non-commercial reuse with attribution permitted.

[4]. Volume 2, Issue 1, January 2012 ISSN: 2277 128X International Journal of Advanced Research in Computer Science and Software Engineering.

[5]. K.H. Bennett et al., "An Architectural Model for Service-Based Software with Ultra Rapid Evolution," Proc.IEEE Int'l Conf. Software Maintenance (ICSM 01),IEEE CS Press, 2001, pp. 292-300.

[6]. Haitham Yaish, Madhu Goyal, George Feuerlicht, "An Elastic Multi-tenant Database Schema for Software as a Service", 2011 Ninth IEEE International Conference on Dependable, Autonomic and Secure Computing.

[7]. Wonjae Lee, Min Choi," A Multi-tenant Web Application Framework for SaaS”, 2012 IEEE Fifth International Conference on Cloud Computing

[8]. Lei Ju, Bikram Sengupta,Abhik Roychoudhury," Tenant Onboarding in Evolving Multi-tenant Software-as-a-Service Systems", 2012 IEEE 19th International Conference on Web Services.

[9]. Krishna Reddy, B. Thirumala Rao, Dr. L.S.S. Reddy and P. Sai Kiran, Research Issues in Cloud Computing, Global Journal of Computer Science and Technology, Vol. 11 No. 11 July 2011

[10] .Krishna Chaitanya.Y, Bhavani Shankar.Y, Kali Rama Krishna.V andV Srinivasa Rao, Study of security issues in Cloud Computing, International Journal of Computer Science and Technology, Vol. 2, No. 3, Sept 2011 